\title{
A sparse regularization approach for ultrafast ultrasound imaging
}

Rafael Carrillo ${ }^{1}$, Adrien Besson ${ }^{13}$, Miaomiao Zhang ${ }^{2}$, Denis Friboulet $^{2}$, Yves Wiaux ${ }^{3}$, Jean-Philippe Thiran ${ }^{1}$ and Olivier Bernard $^{2}$

\author{
${ }^{1}$ Signal Processing Laboratory (LTS5) \\ École Polytechnique Fédérale de Lausanne, Switzerland \\ ${ }^{2}$ CREATIS \\ University of Lyon, France \\ ${ }^{3}$ Institute of Sensors, Signals and Systems \\ Heriot-Watt University, Scotland
}

IEEE International Ultrasonics Symposium, October 2015 


\section{Outline}

Ultrafast Ultrasound Imaging

Principle

A sparse regularization approach to US imaging

The two pillars

The image reconstruction

Experimental study

Protocol

Contrast

Resolution

Conclusions and perspectives 


\section{Outline}

Ultrafast Ultrasound Imaging

Principle

A sparse regularization approach to US imaging

The two pillars

The image reconstruction

Experimental study

Protocol

Contrast

Resolution

Conclusions and perspectives 


\section{Ultrafast Ultrasound Imaging \\ Principle}

Ultrafast Ultrasound imaging 


\section{Ultrafast Ultrasound Imaging \\ Principle}

Ultrafast Ultrasound imaging

- Emission of one single plane wave (PW) or few steered PWs

- Can reach more than thousands frames per second 


\section{Ultrafast Ultrasound Imaging \\ Principle}

Ultrafast Ultrasound imaging

- Emission of one single plane wave (PW) or few steered PWs

- Can reach more than thousands frames per second

- Lower image quality than classical method 


\section{Ultrafast Ultrasound Imaging \\ Principle}

Ultrafast Ultrasound imaging

- Emission of one single plane wave (PW) or few steered PWs

- Can reach more than thousands frames per second

- Lower image quality than classical method

State of the art approaches 


\section{Ultrafast Ultrasound Imaging \\ Principle}

Ultrafast Ultrasound imaging

- Emission of one single plane wave (PW) or few steered PWs

- Can reach more than thousands frames per second

- Lower image quality than classical method

State of the art approaches

- Spatial based approaches: [Montaldo et al., 2009] 
Ultrafast Ultrasound Imaging

Principle

Ultrafast Ultrasound imaging

- Emission of one single plane wave (PW) or few steered PWs

- Can reach more than thousands frames per second

- Lower image quality than classical method

State of the art approaches

- Spatial based approaches: [Montaldo et al., 2009]

- Fourier based approaches: [Lu et al., 1997] [Garcia et al., 2013] [Bernard et al., 2014] 
Ultrafast Ultrasound Imaging

Principle

Ultrafast Ultrasound imaging

- Emission of one single plane wave (PW) or few steered PWs

- Can reach more than thousands frames per second

- Lower image quality than classical method

State of the art approaches

- Spatial based approaches: [Montaldo et al., 2009]

- Fourier based approaches: [Lu et al., 1997] [Garcia et al., 2013] [Bernard et al., 2014]

Ultrasound Fourier slice Beamforming (UFSB) - General scheme

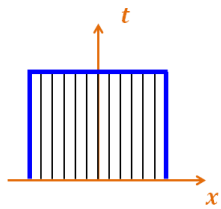


Ultrafast Ultrasound Imaging

Principle

Ultrafast Ultrasound imaging

- Emission of one single plane wave (PW) or few steered PWs

- Can reach more than thousands frames per second

- Lower image quality than classical method

State of the art approaches

- Spatial based approaches: [Montaldo et al., 2009]

- Fourier based approaches: [Lu et al., 1997] [Garcia et al., 2013] [Bernard et al., 2014]

Ultrasound Fourier slice Beamforming (UFSB) - General scheme

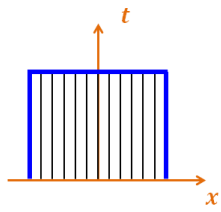

Backscattered echoes

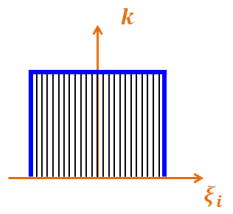

Radial Fourier samples 
Ultrafast Ultrasound Imaging

Principle

Ultrafast Ultrasound imaging

- Emission of one single plane wave (PW) or few steered PWs

- Can reach more than thousands frames per second

- Lower image quality than classical method

State of the art approaches

- Spatial based approaches: [Montaldo et al., 2009]

- Fourier based approaches: [Lu et al., 1997] [Garcia et al., 2013] [Bernard et al., 2014]

Ultrasound Fourier slice Beamforming (UFSB) - General scheme

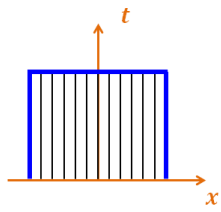

Backscattered echoes

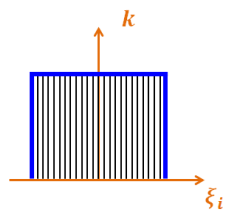

Radial Fourier samples

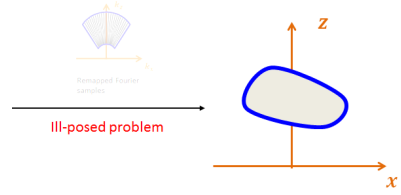

Final image 


\section{Outline}

Ultrafast Ultrasound Imaging

\section{Principle}

A sparse regularization approach to US imaging

The two pillars

The image reconstruction

Experimental study

Protocol

Contrast

Resolution

Conclusions and perspectives 


\section{A sparse regularization approach to US imaging}

The two pillars

UFSB poses an inverse problem 


\section{A sparse regularization approach to US imaging} The two pillars

UFSB poses an inverse problem

$$
\boldsymbol{y}=\Phi \boldsymbol{r}+\boldsymbol{n}, \text { with } \Phi \text { ill-posed }
$$




\section{A sparse regularization approach to US imaging}

The two pillars

UFSB poses an inverse problem

$$
\boldsymbol{y}=\Phi \boldsymbol{r}+\boldsymbol{n}, \text { with } \Phi \text { ill-posed }
$$

- $\boldsymbol{y}$ are the radial Fourier samples, $\boldsymbol{r}$ is the desired image, $\boldsymbol{n}$ is the noise

- Measurement operator: $\Phi$ is the 2D Non-Uniform Fourier Transform 


\section{A sparse regularization approach to US imaging}

The two pillars

UFSB poses an inverse problem

$$
\boldsymbol{y}=\Phi \boldsymbol{r}+\boldsymbol{n}, \text { with } \Phi \text { ill-posed }
$$

- $\boldsymbol{y}$ are the radial Fourier samples, $\boldsymbol{r}$ is the desired image, $\boldsymbol{n}$ is the noise

- Measurement operator: $\Phi$ is the 2D Non-Uniform Fourier Transform

US images are sparse in an appropriate model 


\section{A sparse regularization approach to US imaging}

The two pillars

UFSB poses an inverse problem

$$
\boldsymbol{y}=\Phi \boldsymbol{r}+\boldsymbol{n}, \text { with } \Phi \text { ill-posed }
$$

- $\boldsymbol{y}$ are the radial Fourier samples, $\boldsymbol{r}$ is the desired image, $\boldsymbol{n}$ is the noise

- Measurement operator: $\Phi$ is the 2D Non-Uniform Fourier Transform

US images are sparse in an appropriate model

- Several models already studied: Wavelet basis, Wave atoms frame, Fourier basis 


\section{A sparse regularization approach to US imaging}

The two pillars

UFSB poses an inverse problem

$$
\boldsymbol{y}=\Phi \boldsymbol{r}+\boldsymbol{n}, \text { with } \Phi \text { ill-posed }
$$

- $\boldsymbol{y}$ are the radial Fourier samples, $\boldsymbol{r}$ is the desired image, $\boldsymbol{n}$ is the noise

- Measurement operator: $\Phi$ is the 2D Non-Uniform Fourier Transform

US images are sparse in an appropriate model

- Several models already studied: Wavelet basis, Wave atoms frame, Fourier basis

- Sparsity averaging model (SARA) $\Psi$ used: [Carrillo et al., 2012]

- Concatenation of wavelet basis: $\Psi=\frac{1}{\sqrt{q}}\left[\Psi_{1}, \ldots, \Psi_{q}\right]$

- In the study: $q=8$, Daubechies wavelet as mother function 


\section{A sparse regularization approach to US imaging}

The image reconstruction

Reconstruction problem 


\section{A sparse regularization approach to US imaging}

The image reconstruction

Reconstruction problem

- The image is recovered by solving the inverse problem:

$$
\min _{\overline{\boldsymbol{r}} \in \mathbb{C}^{N}}\left\|\Psi^{H} \overline{\boldsymbol{r}}\right\|_{1} \text { subject to }\|\boldsymbol{y}-\Phi \overline{\boldsymbol{r}}\|_{2} \leq \epsilon
$$

- Non-linear but Convex problem 


\section{A sparse regularization approach to US imaging}

The image reconstruction

Reconstruction problem

- The image is recovered by solving the inverse problem:

$$
\min _{\overline{\boldsymbol{r}} \in \mathbb{C}^{N}}\left\|\Psi^{H} \overline{\boldsymbol{r}}\right\|_{1} \text { subject to }\|\boldsymbol{y}-\Phi \overline{\boldsymbol{r}}\|_{2} \leq \epsilon
$$

- Non-linear but Convex problem

Reconstruction algorithm 


\section{A sparse regularization approach to US imaging}

The image reconstruction

Reconstruction problem

- The image is recovered by solving the inverse problem:

$$
\min _{\overline{\boldsymbol{r}} \in \mathbb{C}^{N}}\left\|\Psi^{H} \overline{\boldsymbol{r}}\right\|_{1} \text { subject to }\|\boldsymbol{y}-\Phi \overline{\boldsymbol{r}}\|_{2} \leq \epsilon
$$

- Non-linear but Convex problem

Reconstruction algorithm

- ADMM algorithm [Boyd et al., 2010]

- Golden section search to find the best value of $\epsilon$ 


\section{Outline}

Ultrafast Ultrasound Imaging

\section{Principle}

A sparse regularization approach to US imaging

The two pillars

The image reconstruction

Experimental study

Protocol

Contrast

Resolution

Conclusions and perspectives 


\section{Experimental study \\ Protocol}

Numerical simulations

- Based on Field II software [Jensen, 1991]

- Contrast to Noise ratio (CNR)

Experimental data

- UlaOp ultrasound scanner with linear probe

- Spatial resolution

Comparisons

- Fourier based approaches: Lu, Garcia and Bernard

- Spatial based approaches: Montaldo 


\section{Experimental study \\ Contrast}

Contrast to Noise Ratio

- Measured from numerical simulations

- $2 \times 2 \mathrm{~cm}$ phantom with high density of scatterers

- $8 \mathrm{~mm}$-diameter anechoic lesion centered inside the phantom 


\section{Experimental study \\ Contrast}

Contrast to Noise Ratio

- Measured from numerical simulations

- $2 \times 2 \mathrm{~cm}$ phantom with high density of scatterers

- $8 \mathrm{~mm}$-diameter anechoic lesion centered inside the phantom

Reconstruction results 


\section{Experimental study}

Contrast

Contrast to Noise Ratio

- Measured from numerical simulations

- $2 \times 2 \mathrm{~cm}$ phantom with high density of scatterers

- 8mm-diameter anechoic lesion centered inside the phantom

Reconstruction results

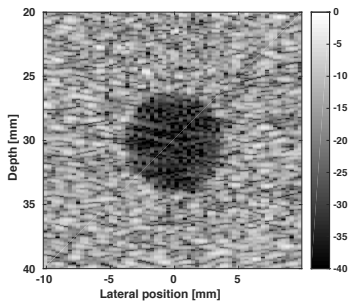

(a) Classic

reconstruction 


\section{Experimental study}

Contrast

Contrast to Noise Ratio

- Measured from numerical simulations

- $2 \times 2 \mathrm{~cm}$ phantom with high density of scatterers

- 8mm-diameter anechoic lesion centered inside the phantom

Reconstruction results

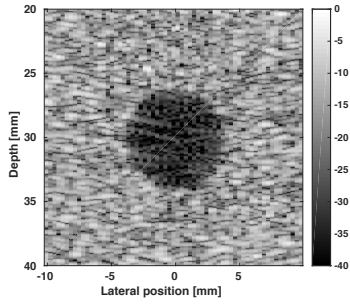

(a) Classic reconstruction

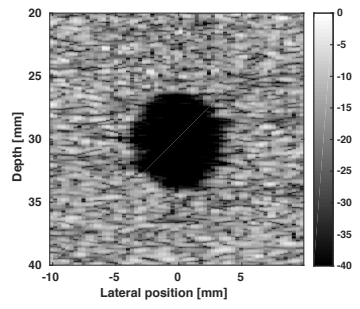

(b) Sparse

reconstruction 


\section{Experimental study}

Contrast

Contrast to Noise Ratio

- Measured from numerical simulations

- $2 \times 2 \mathrm{~cm}$ phantom with high density of scatterers

- $8 \mathrm{~mm}$-diameter anechoic lesion centered inside the phantom

Reconstruction results

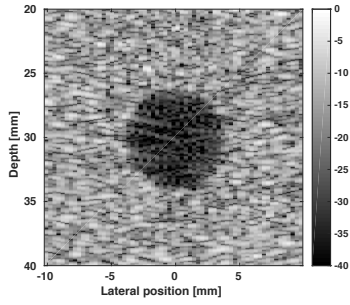

(a) Classic reconstruction

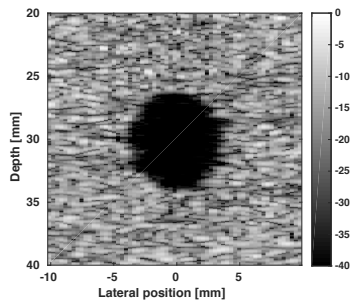

(b) Sparse

reconstruction

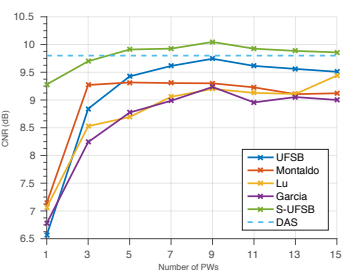

(c) CNR comparison with compounding 


\section{Experimental study}

Spatial Resolution

Experimental data

- Using the UlaOp system with a linear probe (64 elements, $5 \mathrm{MHz}$ center frequency, $50 \mathrm{MHz}$ sampling frequency)

- Measured at different depths 


\section{Experimental study}

Spatial Resolution

Experimental data

- Using the UlaOp system with a linear probe (64 elements, $5 \mathrm{MHz}$ center frequency, $50 \mathrm{MHz}$ sampling frequency)

- Measured at different depths

Reconstruction results 


\section{Experimental study}

Spatial Resolution

Experimental data

- Using the UlaOp system with a linear probe (64 elements, $5 \mathrm{MHz}$ center frequency, $50 \mathrm{MHz}$ sampling frequency)

- Measured at different depths

Reconstruction results 


\section{Experimental study}

Spatial Resolution

Experimental data

- Using the UlaOp system with a linear probe (64 elements, $5 \mathrm{MHz}$ center frequency, $50 \mathrm{MHz}$ sampling frequency)

- Measured at different depths

Reconstruction results

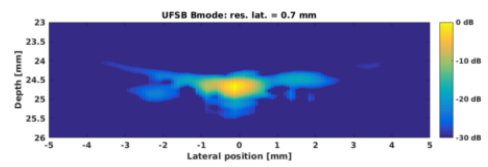

(a) Classic reconstruction 


\section{Experimental study}

Spatial Resolution

Experimental data

- Using the UlaOp system with a linear probe (64 elements, $5 \mathrm{MHz}$ center frequency, $50 \mathrm{MHz}$ sampling frequency)

- Measured at different depths

Reconstruction results

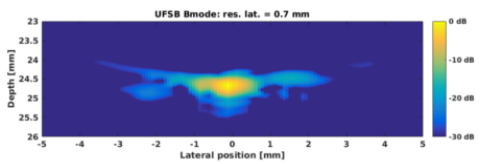

(a) Classic reconstruction

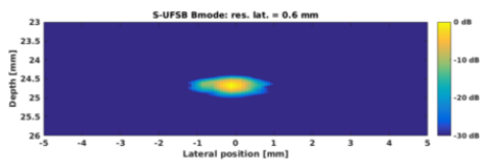

(b) Sparse reconstruction 


\section{Experimental study}

Spatial Resolution

Experimental data

- Using the UlaOp system with a linear probe (64 elements, $5 \mathrm{MHz}$ center frequency, $50 \mathrm{MHz}$ sampling frequency)

- Measured at different depths

Reconstruction results

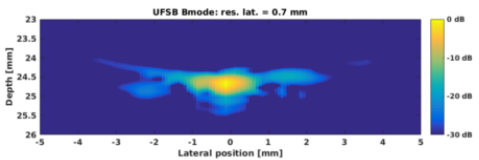

(a) Classic reconstruction

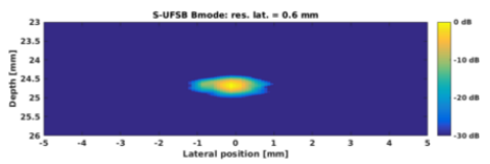

(b) Sparse reconstruction

\begin{tabular}{|c|c|c|c|c|c|c|c|c|c|c|}
\hline \multirow{2}{*}{ Depth } & \multicolumn{2}{|c|}{ Lu } & \multicolumn{2}{c|}{ Garcia } & \multicolumn{2}{c|}{ UFSB } & \multicolumn{2}{c|}{ Montaldo } & \multicolumn{2}{c|}{ S-UFSB } \\
\cline { 2 - 10 } & Axial & Lateral & Axial & Lateral & Axial & Lateral & Axial & Lateral & Axial & Lateral \\
\hline $25 \mathrm{~mm}$ & $0.4 \mathrm{~mm}$ & $0.7 \mathrm{~mm}$ & $0.4 \mathrm{~mm}$ & $0.6 \mathrm{~mm}$ & $0.4 \mathrm{~mm}$ & $0.7 \mathrm{~mm}$ & $0.4 \mathrm{~mm}$ & $0.6 \mathrm{~mm}$ & $0.3 \mathrm{~mm}$ & $0.6 \mathrm{~mm}$ \\
$35 \mathrm{~mm}$ & $0.7 \mathrm{~mm}$ & $0.7 \mathrm{~mm}$ & $0.7 \mathrm{~mm}$ & $0.7 \mathrm{~mm}$ & $0.7 \mathrm{~mm}$ & $0.7 \mathrm{~mm}$ & $0.7 \mathrm{~mm}$ & $0.7 \mathrm{~mm}$ & $0.5 \mathrm{~mm}$ & $0.6 \mathrm{~mm}$ \\
$45 \mathrm{~mm}$ & $0.6 \mathrm{~mm}$ & $1 \mathrm{~mm}$ & $0.6 \mathrm{~mm}$ & $1 \mathrm{~mm}$ & $0.6 \mathrm{~mm}$ & $0.9 \mathrm{~mm}$ & $0.6 \mathrm{~mm}$ & $1 \mathrm{~mm}$ & $0.6 \mathrm{~mm}$ & $1 \mathrm{~mm}$ \\
\hline
\end{tabular}

(c) Spatial resolution from UlaOp scanner 


\section{Outline}

Ultrafast Ultrasound Imaging

\section{Principle}

A sparse regularization approach to US imaging

The two pillars

The image reconstruction

Experimental study

Protocol

Contrast

Resolution

Conclusions and perspectives 


\section{Conclusions and perspectives}

New reconstruction method 


\section{Conclusions and perspectives}

New reconstruction method

- Exploits sparsity of US images to solve the inverse problem 


\section{Conclusions and perspectives}

New reconstruction method

- Exploits sparsity of US images to solve the inverse problem

- Yields better image quality 


\section{Conclusions and perspectives}

New reconstruction method

- Exploits sparsity of US images to solve the inverse problem

- Yields better image quality

- High computational load at reconstruction 


\section{Conclusions and perspectives}

New reconstruction method

- Exploits sparsity of US images to solve the inverse problem

- Yields better image quality

- High computational load at reconstruction

Perspectives 


\section{Conclusions and perspectives}

New reconstruction method

- Exploits sparsity of US images to solve the inverse problem

- Yields better image quality

- High computational load at reconstruction

Perspectives

- Extension to all the Fourier methods (Garcia and Lu) 


\section{Conclusions and perspectives}

New reconstruction method

- Exploits sparsity of US images to solve the inverse problem

- Yields better image quality

- High computational load at reconstruction

Perspectives

- Extension to all the Fourier methods (Garcia and Lu)

- Acceleration and optimization of the current algorithms (GPU implementation) 


\section{Conclusions and perspectives}

New reconstruction method

- Exploits sparsity of US images to solve the inverse problem

- Yields better image quality

- High computational load at reconstruction

Perspectives

- Extension to all the Fourier methods (Garcia and Lu)

- Acceleration and optimization of the current algorithms (GPU implementation)

- Extension of the framework to diverging waves 


\section{Conclusions and perspectives}

New reconstruction method

- Exploits sparsity of US images to solve the inverse problem

- Yields better image quality

- High computational load at reconstruction

Perspectives

- Extension to all the Fourier methods (Garcia and Lu)

- Acceleration and optimization of the current algorithms (GPU implementation)

- Extension of the framework to diverging waves 
THANK YOU FOR YOUR ATTENTION! 


\section{ADMM algorithm}

- The general problem we solve is the following one:

$$
\min _{\overline{\overline{\boldsymbol{x}}} \in \mathbb{C}^{N}} f(\overline{\boldsymbol{x}}) \text { subject to } h(\boldsymbol{y}-\Phi \overline{\boldsymbol{x}})=0,
$$

- The ADMM algorithm is:

Input: $\mathrm{k}=0$, choose $\boldsymbol{x}^{\mathbf{0}}, \boldsymbol{z}^{\mathbf{0}}, \boldsymbol{\lambda}^{\mathbf{0}}, \mu>0, \gamma>0$

1: repeat

2: $\quad \boldsymbol{z}^{(t+1)}=\operatorname{prox}_{\gamma h}\left(\boldsymbol{y}-\Phi \boldsymbol{x}^{(t)}-\boldsymbol{\lambda}^{(t)}\right)$

3: $\quad \boldsymbol{x}^{(t+1)}=\operatorname{prox}_{\mu \gamma f}\left(\boldsymbol{x}^{(t)}-\mu \Phi^{H}\left(\boldsymbol{\lambda}^{(t)}+\Phi \boldsymbol{x}^{(t)}-\boldsymbol{y}+\boldsymbol{z}^{(t+1)}\right)\right)$

4: $\quad \boldsymbol{\lambda}^{(t+1)}=\boldsymbol{\lambda}^{(t)}+\beta\left(\Phi \boldsymbol{x}^{(t+1)}-\boldsymbol{y}+\boldsymbol{z}^{(t+1)}\right)$

5: until A stopping criterion is met 